\title{
12. ON THE NATURE OF PLANETARY NEBULAE
}

\author{
I. S. SHKLOVSKY \\ Sternberg Astronomical Institute, Moscow, U.S.S.R.
}

The physical state of the planetary nebulae has been investigated sufficiently well, but the main question of their origin and evolution still remains open.

For the solution of this most significant problem, it is very important in our opinion to analyse the well-known fact that the planetary nebulae undergo an unlimited expansion, and a consequent dissipation. According to obseryations, the velocities of expansion of the planetary nebulae equal several tens of kilometres per second. The same velocity should also be expected when hot and comparatively dense gases expand into a vacuum.

Two principal conclusions may be drawn from the fact of infinite expansion of planetary nebulae: (a) the optical thickness of each planetary nebula decreases continuously until it becomes less than unity. (b) The luminosity of an optically thin planetary nebula decreases with the evolution of the nebula approximately as $r^{-3}$, which is proportional to $t^{-3}$, where $r$ is the radius of the nebula and $t$ is the time. The surface brightness decreases approximately as $r^{-5}$, which is proportional to $t^{-5}$.

The brightness of the majority of planetary nebulae will, over several thousands of years, decrease to such an extent that they will no longer be observable. It follows then that the process of continuous formation of planetary nebulae is going on in the Galaxy. The number of planetary nebulae formed every year in our Galaxy is of the order of unity.

No adequate methods exist at the present time for the determination of the distances of the planetary nebulae. It can be shown that the wellknown methods of Vorontsov-Velyaminov, Berman, and Camm cannot be considered as correct. For example, the main assumption in VorontsovVelyaminov's method that all planetary nebulae have the same luminosity is not correct, because as a result of their expansion, the luminosities of the planetary nebulae decrease indefinitely. The young, star-like planetary nebulae must possess the highest luminosities and very high surface brightnesses. The luminosities of the old, greatly expanded objects with low surface brightnesses must be the lowest. 
In accordance with these ideas, we have developed a new method for the determination of the distances of the planetary nebulae, based upon physical reasoning. From simple reasoning, it follows that the distance of an optically thin planetary nebula is

$$
R \propto \frac{\mathbf{m}^{0 \cdot 4}}{\phi I^{0 \cdot 2}},
$$

where $\mathbf{m}$ is the mass, $I$ the surface brightness, and $\phi$ the angular dimensions. Estimates of the distance depend but very little upon the distribution of the surface brightness, because $R$ depends to a very small extent upon $I$. A new catalogue of planetary distances has been computed. The masses of planetary nebulae have been considered for this purpose to be equal. This assumption cannot influence the results seriously, because $R$ depends but slightly upon $\mathbf{m}$. The zero-point of the new system of distances was determined from an analysis of the known proper motions and radial velocities, the interstellar absorption being taken into account. The newly derived distances differ greatly from the previously accepted values. Thus, for instance, NGG 7293 has a distance of $5^{\circ}$ parsecs in the new catalogue, while according to Vorontsov-Velyaminov it is 250 parsecs, and according to Berman it equals I050 parsecs.

It should be mentioned that Van Maanen determined a parallax of $0 " 038 \pm 0 " 008$ for the nucleus of NGG 7293 ; this fact was, however, not taken into account in the present analysis. It may be shown that if NGG 7293 had a distance exceeding $25^{\circ}$ parsecs, its mass should be larger than Io m॰. Such a value is hardly acceptable. For $R=50$ parsecs, the mass would be about $0 \cdot 2$ m॰.

An analysis of the new system of distances permits one to draw some conclusions about the absolute magnitudes of the nuclei of planetary nebulae. The nebulae themselves show an extremely large scatter, ranging from $M=-0.5$ to +10 . The absolute magnitudes of a number of nuclei of planetary nebulae range from $M=+5$ to + Io. As the temperatures of the nuclei are extremely high, it may easily be shown that these stars possess mean densities similar to those of white dwarfs. Consequently, the nuclei of a number of planetary nebulae are 'over-heated' white dwarfs.

We believe such a conclusion is of particular importance in a consideration of the planetary nebulae. After some tens of thousands of years, the dimensions of the nebula NGC 7293 will increase several times. Its brightness also must diminish by a factor of several hundred, and the nebula will become invisible. Only the nucleus will remain as an extremely hot white dwarf. The nucleus will cool and change gradually into a normal 
white dwarf. The absolute magnitudes of the majority of nuclei of planetary nebulae considerably exceed the absolute magnitudes of white dwarfs. This fact may indicate that along with a rapid evolution of planetary nebulae, there also takes place a rather rapid (but not an explosive) evolution of their nuclei, the luminosity and temperature of the nuclei thereby decreasing. This possibility may explain the well-known fact that the spectral characteristics of the various nuclei correspond to different morphological types of the associated nebulae. The genetic connexion between planetary nebulae and white dwarfs suggests that some stars at a definite stage of their evolution detach a shell with zero velocity in the process of transformation into white dwarfs. This process would go on for several tens of thousands of years.

Great theoretical difficulties arise if such an interpretation is accepted, but they do not seem insurmountable to us.

It is important to note that the creation of the planetary nebulae from stars is not a recurrent process. This conclusion follows from the value of the mass of planetary nebulae ( $\mathbf{m}$ about $0.2 \mathrm{~m} \odot$ ).

Several billions of planetary nebulae have formed and then decayed during the life-period of the Galaxy. It has recently been estimated that the number of white dwarfs existing in our stellar system is about the same number. This is an independent confirmation of our proposal.

The continuous process of formation of planetary nebulae is the most powerful supplier of gas to interstellar space. A mass of about several tenths of one solar mass per year appears in the Galaxy due to this mechanism. This exceeds by several hundred times the amount of mass ejected by all the novae.

As the luminosity of the planetary nebulae decreases, their optical thickness beyond the Lyman limit becomes very small. All recent methods for the determination of the temperatures of planetary nebulae gave a much underestimated value of $T_{*}$. The large abundance of hydrogen in the planetary nebulae is of interest for the theory of white dwarfs.

It is important to establish the nature of stars that create the planetary nebulae. If the development is extrapolated back to the early stages of expansion, the stars must have been objects of high luminosity. The nebular lines in their spectra must then have been fainter than the hydrogen lines and other permitted lines, because the electron concentration was then relatively high. The optical thickness beyond the Lyman limit must have been much greater than unity. Such objects must possess quite bright continua, which originate from two-photon transitions from the ${ }^{2} S$ level of hydrogen. Objects similar to planetary nebulae, but with 
spectra similar to some Be stars, have been observed by Dr Minkowski. It is clear, however, that unrestricted extrapolation is not safe in this case.

Although the conditions prevailing in the earliest stages of the formation of planetary nebulae are not known, one has the impression that the immediate predecessors of the planetary nebulae may be some type of peculiar red giant star of high luminosity. It is probable that such stars are known, but there is as yet no suspicion that these are the 'proto-planetary nebulae'. These peculiar stars of high luminosity must be a definite, regular, but rather short-lived stage in the evolution of an extremely numerous type of star. It is natural to connect our suggestion with the theory of stellar evolution that has been developed by Schwarzschild and others.

Special observations may prove and extend these considerations. A new determination of the trigonometric parallax of NGG 7293 would be particularly important.

\section{BIBLIOGR A PHY}

I. S. Shklovsky, A.J.U.S.S.R. 33, 222, 315 (1956). 